\title{
Imaging the Biodistribution and Performance of Transplanted Stem Cells with PET
}

\author{
Christopher G. England ${ }^{1}$, Emily B. Ehlerding ${ }^{1}$, and Weibo $\mathrm{Cai}^{1-3}$ \\ ${ }^{1}$ Department of Medical Physics, University of Wisconsin-Madison, Madison, Wisconsin; ${ }^{2}$ Department of Radiology, University of \\ Wisconsin-Madison, Madison, Wisconsin; and ${ }^{3}$ Carbone Cancer Center, University of Wisconsin, Madison, Wisconsin
}

$\mathbf{S}$ stem cells for treatment of muscle-related diseases (1), yet clinical translation of these cell-based therapies has been limited by the host immune response, ineffective homing strategies for getting the donor cells to targeted musculature, and the intricate generation of large numbers of transplantable cells. Because highly invasive tissue biopsies are often required to diagnose muscle-related diseases and monitor their treatment, there is a dire need for alternative noninvasive approaches. Recently, PET imaging of biomarkers has shown great potential for tracking disease progression and monitoring therapeutic interventions. More specifically, PET imaging can track injected cells of interest that have been directly labeled with an imaging tag or indirectly labeled with a reporter, allowing for in vivo tracking of their fate. This ability virtually eliminates the need for highly invasive procedures, in addition to providing fast and reliable data.

\section{See page 1467}

In this issue of The Journal of Nuclear Medicine, Haralampieva et al. from ETH Zurich report on the noninvasive PET imaging of human muscle precursor cells (hMPCs) in a nude mouse model using a signaling-deficient human dopamine $\mathrm{D}_{2}$ receptor $\left(\mathrm{hD}_{2} \mathrm{R}\right)$ as a reporter for binding of ${ }^{18} \mathrm{~F}$-fallypride (2). There were 3 distinct reasons why the authors chose $\mathrm{hD}_{2} \mathrm{R}$, which has been extensively studied as the reporter gene. First, the fact that $\mathrm{hD}_{2} \mathrm{R}$ is normally expressed only in the striata nigra simplifies tracking of transplanted $\mathrm{hD}_{2} \mathrm{R}$-expressing cells in peripheral body regions, whose native tissues do not express $\mathrm{hD}_{2} \mathrm{R}$. Second, $\mathrm{hD}_{2} \mathrm{R}$ may be genetically mutated to eliminate the potential activation of signaling transduction pathways on binding of $\mathrm{hD}_{2} \mathrm{R}$ ligands. Normally, binding of $\mathrm{hD}_{2} \mathrm{R}$ by its native ligands results in activation of a G-protein signaling pathway; thus, the activity of this receptor needs to be silenced to ensure that the genetically altered hMPCs display the

Received Mar. 9, 2016; revision accepted Apr. 11, 2016.

For correspondence or reprints contact: Weibo Cai, University of WisconsinMadison, Room 7137, 1111 Highland Ave., Madison, WI 53705.

E-mail: wcai@uwhealth.org

Published online May 19, 2016.

COPYRIGHT (C) 2016 by the Society of Nuclear Medicine and Molecular Imaging, Inc.

DOI: $10.2967 /$ jnumed.116.173963 same phenotypic properties as normal hMPCs (3). Genetic uncoupling of the binding activity from the intracellular signaling of $\mathrm{hD}_{2} \mathrm{R}$ prevents the activity of hMPCs from being affected by binding of an endogenous ligand or targeted imaging agent. Third, there are several highly efficient PET imaging agents already available for imaging $\mathrm{hD}_{2} \mathrm{R}$.

Next, the authors chose a transfection method for inserting this mutated gene into the hMPCs. An adenoviral construct was used to transfect cells with the mutated $\mathrm{hD}_{2} \mathrm{R}$ gene. After transfection, $\mathrm{hD}_{2} \mathrm{R}$-transfected cells showed no signs of toxicity and the proliferation rate of transfected hMPCs remained unchanged. Together, these data verified the simplicity and high efficiency of the adenoviral system for rapid transfection of the $\mathrm{hD}_{2} \mathrm{R}$ gene into hMPCs.

Afterward, the authors assessed the binding affinity of $\mathrm{hD}_{2} \mathrm{R}$ hMPCs for ${ }^{18} \mathrm{~F}$-fallypride in vitro. ${ }^{18} \mathrm{~F}$-fallypride is a high-affinity $\mathrm{D}_{2} / \mathrm{D}_{3}$ antagonist radioactive tracer used for PET imaging of extrastriatal dopamine receptors in low concentrations (4). Also, the pharmacokinetic properties and efficacy of ${ }^{18} \mathrm{~F}$-fallypride have been extensively investigated, making it an optimal candidate for imaging of transplanted $\mathrm{hD}_{2} \mathrm{R}$ hMPCs. Because the authors did not provide a rationale for choosing ${ }^{18} \mathrm{~F}$-fallypride in this study, we predict that future studies may compare other commonly used $\mathrm{hD}_{2} \mathrm{R}$ based PET tracers, including ${ }^{124} \mathrm{I}$-epidepride, ${ }^{76} \mathrm{Br}$-isoremoxipride, and perhaps their ${ }^{11} \mathrm{C}$-labeled derivatives for tracking the biodistribution of $\mathrm{hD}_{2} \mathrm{R}$ hMPCs transplanted in vivo (5).

To track the fate of hMPCs in vivo, the authors subcutaneously injected nude mice with transfected $\mathrm{hD}_{2} \mathrm{R}$ hMPCs before performing PET imaging with ${ }^{18} \mathrm{~F}$-fallypride. High uptake of ${ }^{18} \mathrm{~F}$-fallypride in $\mathrm{hD}_{2} \mathrm{R}$ hMPCs was seen at 1 wk after transplantation, whereas uptake was significantly lower at $2 \mathrm{wk}$. The authors attributed this signal decrease to the posttranslational modification of ectopically expressed $\mathrm{hD}_{2} \mathrm{R}$ or the internalization and degradation of $\mathrm{hD}_{2} \mathrm{R}$ during myofibril differentiation. Low PET signal in the cerebellum further confirmed the high specificity of ${ }^{18} \mathrm{~F}$-fallypride for $\mathrm{hD}_{2} \mathrm{R}$ hMPCs. In addition, PET studies were performed using haloperidol as an $\mathrm{hD}_{2} \mathrm{R}$-blocking agent: $\mathrm{hD}_{2} \mathrm{R}$-expressing cells and tissues showed decreased PET signal after the injection of haloperidol. Next, the authors investigated the oxygenation status of transplanted $\mathrm{hD}_{2} \mathrm{R}$ hMPCs by PET imaging with ${ }^{18} \mathrm{~F}$-fluoromisonidazole. Uptake of ${ }^{18}$ F-fluoromisonidazole was projected to be highest soon after cell transplantation, as low oxygen levels are found during the initial stages of cell-to-myofibril formation. ${ }^{18} \mathrm{~F}$-fluoromisonidazole is the most widely investigated PET tracer for preclinical and clinical imaging of hypoxia. As expected, uptake of ${ }^{18} \mathrm{~F}$-fluoromisonidazole was relatively high at $1 \mathrm{wk}$ after transplantation, and this high 
uptake was further corroborated by increased protein levels of the endothelial cell marker vWf (Von Willebrand factor) and elevated vascular endothelial growth factor-A gene expression at later time points. Together, increased vascular endothelial growth factor-A and endothelial cell markers, in combination with high oxygen consumption, indicate actively growing tissues. The authors concluded that ${ }^{18} \mathrm{~F}$-fluoromisonidazole allowed for sensitive monitoring of hypoxia-related biologic processes in bioengineered constructs.

Although this study provided a methodology for tracking hMPCs in vivo, this technology can be further implemented to track the fate of other transplanted cells. For example, Schönitzer et al. reported a similar approach for noninvasively tracking human mesenchymal stem cells using a different mutated form of $\mathrm{hD}_{2} \mathrm{R}$ (6). Only $25 \%$ of the cells showed successful transfection of $\mathrm{hD}_{2} \mathrm{R}$ in the hMPCs; other transfection strategies may provide better transfection rates. In addition, reporter genes can complicate PET quantification and in vivo kinetic modeling of imaging tracers. Effective tracer uptake is dependent on the availability of targeted cells, the injected tracer dose, the regional blood flow and vascularity of the tissue, and the pharmacokinetics of tracer uptake (7). It was previously described that reporter genes encoding for intracellular enzymes or transporters show superiority over membrane receptors such as $\mathrm{hD}_{2} \mathrm{R}$ because they allow the tracer to accumulate inside the cell, whereas binding to surface receptors may be weak and reversible ( 8 ). In addition to the dopamine model described in this study, the sodium iodide symporter transfection model is an alternative that has been commonly used for PET and SPECT imaging (9).

The clinical applicability of stem cell-based therapies is slowly becoming a potential reality. The use of cell-based therapies for the treatment of muscle-related diseases is promising, yet many uncertainties remain. First, transfected stem cells have been widely explored in basic preclinical research, yet these transfection models have been limited to cell imaging and not therapeutic intervention. For this reason, clinical use of reporter gene systems for patient treatment remains unlikely at this time. Currently, ${ }^{18} \mathrm{~F}-\mathrm{FDG}$ is the only PET tracer used in a clinical setting for stem cell tracking (10). Also, reporter gene models may be limited by changes in the expression of the transfected gene and encoded receptor. Additionally, the effects of the host immune system on the efficacy of cell-based therapies remain unclear. In the future, dual treatment strategies using both gene- and cell-based therapies may show additive or synergistic therapeutic effects in comparison to monotherapy-based approaches. As new cell-based therapeutic strategies are investigated, the noninvasive high-specificity tracking of transplanted cells using PET or other imaging modalities may eliminate the necessity for highly invasive procedures. As researchers continue to investigate novel cell-based therapies, molecular imaging must continue to evolve to fill the need for noninvasive imaging approaches. This field holds great promise, and we expect that cell-based therapies will become a vital part of personalized medicine for the treatment of a broad range of diseases.

\section{DISCLOSURE}

This work was supported, in part, by the University of Wisconsin-Madison, the National Institutes of Health (NIBIB/ NCI 1R01CA169365, P30CA014520, T32CA009206, and T32GM008505), and the American Cancer Society (125246-RSG13-099-01-CCE). No other potential conflict of interest relevant to this article was reported.

\section{REFERENCES}

1. Skuk D. Myoblast transplantation for inherited myopathies: a clinical approach. Expert Opin Biol Ther. 2004;4:1871-1885.

2. Haralampieva D, Betzel T, Dinulovic I, et al. Noninvasive PET imaging and tracking of engineered human muscle precursor cells for skeletal muscle tissue engineering. J Nucl Med. 2016;57:1467-1473.

3. Boyd KN, Mailman RB. Dopamine receptor signaling and current and future antipsychotic drugs. Handb Exp Pharmacol. 2012;212:53-86.

4. Vandehey NT, Moirano JM, Converse AK, et al. High-affinity dopamine D2/D3 PET radioligands ${ }^{18} \mathrm{~F}$-fallypride and ${ }^{11} \mathrm{C}$-FLB457: a comparison of kinetics in extrastriatal regions using a multiple-injection protocol. J Cereb Blood Flow Metab. 2010;30:994-1007.

5. de Paulis T. The discovery of epidepride and its analogs as high-affinity radioligands for imaging extrastriatal dopamine $\mathrm{D}_{2}$ receptors in human brain. Curr Pharm Des. 2003;9:673-696.

6. Schönitzer V, Haasters F, Kasbauer S, et al. In vivo mesenchymal stem cell tracking with PET using the dopamine type 2 receptor and ${ }^{18} \mathrm{~F}$-fallypride. $J$ Nucl Med. 2014;55:1342-1347.

7. Terrovitis JV, Smith RR, Marban E. Assessment and optimization of cell engraftment after transplantation into the heart. Circ Res. 2010;106:479-494.

8. Chen IY, Wu JC, Min JJ, et al. Micro-positron emission tomography imaging of cardiac gene expression in rats using bicistronic adenoviral vector-mediated gene delivery. Circulation. 2004;109:1415-1420.

9. Chung J-K. Sodium iodide symporter: its role in nuclear medicine. J Nucl Med. 2002;43:1188-1200.

10. Kooreman NG, Ransohoff JD, Wu JC. Tracking gene and cell fate for therapeutic gain. Nat Mater. 2014;13:106-109. 